\title{
Carnets
}

Revue électronique d'études françaises de l'APEF

Première Série - 2 Numéro Spécial 10-11 | 2011

D'un Nobel l'autre

\section{D'un nobel l'autre : Mutations culturelles et évolutions esthétiques de la littérature narrative en France}

Bruno Blanckeman

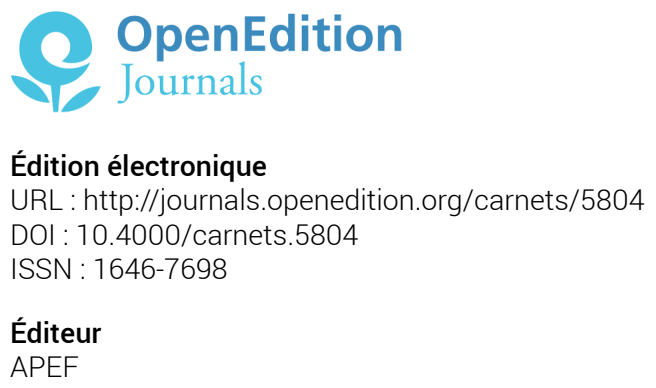

Édition imprimée

Date de publication : 1 janvier 2011

Pagination : 257-265

Référence électronique

Bruno Blanckeman, «D'un nobel l'autre : Mutations culturelles et évolutions esthétiques de la littérature narrative en France », Carnets [En ligne], Première Série - 2 Numéro Spécial 10-11 | 2011, mis en ligne le 16 juin 2018, consulté le 24 septembre 2020. URL : http://journals.openedition.org/carnets/ 5804 ; DOI : https://doi.org/10.4000/carnets.5804

\section{(9) $(1) \Theta$}

Carnets est mis à disposition selon les termes de la licence Creative Commons - Atribution - Pas d'utilisation commerciale 4.0 International. 


\section{D'UN NOBEL L'AUTRE}

\section{Mutations culturelles et évolutions esthétiques de la littérature narrative}

\section{en France}

BRUNO BLANCKEMAN

Université de la Sorbonne Nouvelle Paris III bruno.blanckeman@univ-paris3.fr 
De 1985 à 2008, d'un Nobel l'autre, la durée, même relative, permet de prendre la mesure du devenir culturel et esthétique de la littérature, ses mutations à l'orée d'un siècle nouveau, mais aussi ses éléments de continuité. Une page d'histoire conséquente se tourne, celle de la modernité et de ses innombrables arabesques, ce qui ne signifie toutefois pas qu'elle s'efface, le temps à bien des égards cyclique du littéraire ne se ramenant pas à celui, calendaire, de l'histoire. Si personne ne songe à rapprocher les oeuvres de Claude Simon et de Le Clézio, nul ne saurait toutefois ignorer ce que leur itinéraire d'écrivain possède en commun. C'est que l'un et l'autre appartiennent à une même ordre de littérature, celui dont justement on peut dire qu'à l'échelle des siècles il fait la littérature elle-même, la fait avancer, fût-ce en en malmenant les formes. L'un et l'autre sont entrés par la force en littérature, opposant aux formes fixes sclérosant l'écriture et la pensée une poétique de la fracture, marquée dans les deux cas mais avec un décalage d'une génération, par l'ombre tutélaire du Nouveau Roman et, plus généralement, par la veine expérimentale du roman moderne. Passé le temps de cette regénération musclée des pratiques narratives, chacun d'eux, l'un plus que l'autre, a évolué vers une écriture romanesque pacifiée, aucun ne se satisfaisant des jeux virtuoses de la littérature formaliste ou des apories nihilistes de la déconstruction. C'est donc avant tout parce que l'un et l'autre s'affirment à des degrés différents comme des explorateurs de formes que leurs oeuvres respectives non seulement stimulent l'imaginaire mais aussi polarisent les consciences autour de certains enjeux majeurs débordant la seule littérature. Claude Simon est l'un des principaux écrivains mémorialiste et penseur de ces deux guerres mondiales qui clivèrent le XXe siècle et en constituent aujourd'hui les archives à charge; Le Clézio, l'un des premiers écrivains moralistes d'un fin et d'un tournant de siècle dont il pointe, en termes de civilisation, de rapport à autrui et d'intégration dans un milieu ambiant, les aberrations quelque peu suicidaires.

C'est cela, me semble-t-il, que consacrent à distance - une page dans le XXe siècle, une autre dans le XXle - ces deux Prix. Une oeuvre, certes, mais surtout à travers elle, une certaine conception de la littérature, telle qu'elle éprouve les formes de la langue, du récit, de la fable, c'est-à-dire notre façon de penser le monde, et une certaine représentation du rôle de l'écrivain dans la cité, tel qu'il s'affirme comme mémoire de la communauté et conscience critique de son temps. Considérations esthétiques et considérations politiques interfèrent en l'occurence dans l'attribution de cette dignité que représente un Nobel. C'est donc aux confins de l'une et de l'autre, ce qui fait de la littérature à la fois un art et une discipline, un lieu de création spécifique mais aussi un foyer intellectuel au sens large que se situera mon approche: qu'en est-il aujourd'hui de la littérature, et plus particulièrement de la littérature narrative en France? quelle place est la sienne dans la société, quel est son champ d'action public, quels discours d'escorte l'accompagnent? mais quels horizons imaginaires, quelles 
orientations esthétiques la caractérisent, au travers desquels certains écrivains agissent sur leur époque en même temps qu'ils aident à la représenter?

Plusieurs générations d'écrivains confèrent aujourd'hui à la littérature française une vitalité réjouissante. C'est la génération des grands aînés, écrivains sexagénaires, sinon septuagénaires, dont l'oeuvre en voie de classicisation, à l'image de celle de Le Clezio, se poursuit depuis plusieurs décennies: Patrick Modiano, Pascal Quignard, Jean Échenoz, Pierre Michon, Annie Ernaux, Pierre Guyotat, Assia Djebar, Philippe Sollers, Olivier Rolin, François Bon entre autres. C'est la génération des quadra- et quinquagénaires dont on commence à célébrer les oeuvres comme majeures pour certains, même sur le mode de la controverse - Michel Houellebecq, Sylvie Germain, Marie Ndiaye, Patrick Chamoiseau, Laurent Mauvignier -, ou qui peinent encore à se faire pleinement reconnaître malgré leur réelle puissance - Antoine Volodine, Éric Chevillard, Linda Lê, Régine Detambel, Nicole Caligaris. C'est la génération nouvelle, celle de trentenaires sur lesquels, à côté des seuls coups médiatiques, on peut tenter un pari: Tanguy Viel, Chloé Delaume, Pierre Senges, par exemple. Des oeuvres d'intérêt se distinguent ainsi de la production de masse en cela qu'elles travaillent les formes littéraires, entre autres dans les domaines magnétiques de la fiction romanesque et des récits de soi, ouverts sans tabous, sans interdits esthétiques, à l'exploration de l'histoire collective comme aux postulations de la mémoire individuelle, à la représentation des identités culturelles autant qu'aux énigmes de la personnalité intime.

Et pourtant il est un paradoxe foncier quand on parle aujourd'hui de la littérature en France: le discours qui prévaut est celui de la déploration. Critiques et essayistes de JeanMarie Domenach - Le Crépuscule de la culture française, 1995 - à Jean Bothorel - Chers imposteurs, 2008 - via Tzevan Todorov - La Littérature en péril, 2007 - entonnent à intervalles réguliers un refrain qui, avec ses quatre couplets progressifs, constitue un seul et même chant du déclin. Premier couplet: le dépérissement du roman - on le disait déjà à l'époque où Proust achevait La Recherche, il est plaisant de l'entendre encore alors que Le Clézio reçoit le Nobel. Deuxième couplet, où l'on passe d'un genre à la discipline même dont il relève, la disparition de la littérature, son invisibilité culturelle - et pourtant, les lieux de rencontre, de manifestations, de débats, de signatures, de conférences, de résidences n'ont cessé en vingt ans de se multiplier. Troisième couplet, où l'on passe de cette discipline à la sphère civile dans laquelle elle s'inscrit et dont elle constitue l'emblème, la mort de la culture française - formule qui fit la une du Times Magazine en 2007. Quatrième et dernier couplet, où l'on passe de cette sphère civile aux figures qui l'incarnent: le silence des intellectuels, dénoncé par Elisabeth Badinter dès 1983 dans un article du "Monde", leur absence de la scène publique, et s'ils se taisent, s'ils font défaut, c'est peut-être tout simplement parce qu'ils sont morts avec les années 1980 (Sartre, Barthes, Aragon, Lacan, Beauvoir). Ce procès s'apparente en quelque sorte à une nouvelle trahison des clercs dont les termes 
inverseraient ceux formulés à la fin des années 1920 par Julien Benda à l'encontre des écrivains les plus illustres de son temps, dans son célèbre pamphlet ainsi intitulé. Pour Benda, les intellectuels des années 1920 auraient trahi leur vocation de clercs en délaissant l'univers éthéré des principes atemporels et des valeurs universelles, en devenant des intellectuels engagés, en prenant parti ou s'affiliant à un parti. Nouvelle trahison des clercs aujourd'hui, inversée: les écrivains et intellectuels préfèreraient à toute prise de position idéologique, à toute forme d'intervention politique le huis clos d'une Tour d'ivoire relookée en quelque clinquant plateau médiatique. Ce chant du déclin n'est pas le privilège des seuls critiques et l'on connaît quelques écrivains - et non des moindres, comme Richard Millet qui l'inscrivent à leur répertoire, façon pour eux de camper dans la posture prestigieuse du dernier des Mohicans.

Deux questions se posent alors: pourquoi une telle vision des choses et de quoi ce discours négativiste est-il le symptôme? comment, à l'encontre de ce constat d'impuissance, se manifestent aujourd'hui à même les oeuvres une action, sinon un activisme, de l'écrivain, et dans quels domaines propres à intéresser la chose publique?

Le sentiment d'une défection de la littérature, d'une mise sur la touche des écrivains résulte en fait de la normalisation de la place qui leur est accordée dans une société qui les a pendant plusieurs siècles valorisés à l'extrême. En ce sens, c'est moins ce processus actuel qui devrait surprendre que la surdétermination antérieure du rôle public de la littérature en France. En d'autres termes, on assiste depuis une trentaine d'années à un phénomène de désacralisation du littéraire: une banalisation de l'objet-livre depuis l'entrée dans une société dite de consommation, une relativisation de la figure culturelle de l'écrivain avec l'entrée dans une société dite du spectacle. Ce sont ces deux évolutions de société conjointes qui suscitent l'impression que la littérature, tenue pour l'expression-culte du génie français, et l'écrivain, considéré comme le maître à penser de l'intelligence nationale, se sont évanouis dans la nuit des temps - d'où l'attitude qui consiste à déplorer d'une part, à stigmatiser de l'autre. La littérature est aussi devenue une branche annexe de l'industrie des loisirs, sans cesser d'être pour autant un mode ambitieux de création, l'écrivain est devenu un acteur social parmi d'autres, sans cesser pour autant de participer à la vie publique: fin d'une certaine exception française en la matière, historiquement déterminée donc nécessairement transitoire, le plus étonnant étant peut-être qu'elle ait pu durer à peu près quatre siècles. La sacralisation du livre ne fut pas en effet un vain mot: elle s'est marquée à l'origine par la synonymie entre le livre et le Livre, la Bible, puis au fil des siècles par les différents substrats qui investirent l'objet livre d'une valeur transcendante, qu'elle s'identifie, au dix-septième siècle, à la vérité révélée des classiques, qu'elle se convertisse au dix-huitième siècle en un absolu laïc et génère une bible des temps modernes, l'Encyclopédie, ou qu'elle consacre à partir du dix-neuvième siècle un genre littéraire alors tenu pour mineur, le roman, qui se 
légitime lui-même comme un univers conçu, ordonné, créé à l'image du nôtre par quelque romancier, démiurge et omniscient, à l'image de Dieu, donc, et cela à des fins de révéler des vérités universelles. De Proust à Claude Simon, de Roussel à Quignard, les modernes ont moins renoncé à ce modèle qu'ils n'ont joué de façon plus ou moins dissonante avec lui et en ont entretenu la nostalgie, dans des formes sphériques plus ou moins piégées. Quant à la surdétermination du rôle de l'écrivain dans la cité française, elle commence dès la Renaissance, lorsqu'un monarque éclairé - François $1^{\mathrm{er}}$ - coopère avec des poètes pour forger de toute pièces une langue nationale alors inexistante, le français, alors concurrencé à l'écrit par le latin, langue savante, à l'oral par les multiples dialectes. On sait comment un siècle plus tard, avec la Monarchie absolue, cette reconnaissance publique de l'écrivain s'institutionnalise, devient une mission d'intérêt général en même temps que la littérature une académie, qu'il convient d'encadrer pour qu'elle puisse à son tour cadrer les pratiques et diffuser les valeurs, qu'elle fonctionne comme un diffuseur idéologique central. À partir du XVIIIe siècle, c'est toute la capacité de la littérature à s'ériger comme contre-pouvoir à part entière qui s'instaure, d'abord avec la figure du philosophe des Lumières, puis, du Romantisme à Tel Quel, avec la figure de l'écrivain d'avant-garde, enfin, d'une fin de siècle à l'autre et de Zola à Sartre, avec la figure de l'intellectuel engagé. C'est la fin d'une telle hypostasie culturelle - celle du livre, celle de l'écrivain - qui explique en partie la nostalgie que trahit le discours sur la mort de la littérature et la désertion des écrivains. À la base de ce discours il est donc un contresens: un phénomène de relativisation culturelle est interprété comme un phénomène de déperdition esthétique et intellectuel - une perte générale de la valeur des oeuvres - mais aussi politique - une perte collective de l'intérêt des écrivains pour le débat d'idées et le combat des valeurs.

Peut-être s'agit-il alors de déshistoriciser notre vision de la littérature pour mieux être en mesure de la repolitiser. La déshistoriciser: ne plus la penser de façon idéalisatrice comme cette institution qui a longtemps fonctionné comme instance symbolique centrale, comme espace-modèle de la vie publique. La repolitiser, au sens premier du mot politique, comprendre comment peuvent agir les écrivains dans la Cité aujourd'hui, quelles nouvelles modalités d'intervention sont les leurs depuis leur maîtrise des formes littéraires, quelles nouvelles figures d'autorité et quelles pratiques à elles associées, quelles nouvelles mythologies aussi peut-être, se mettent en place. Ainsi, par exemple, de l'idée d'engagement: sous ses formes anciennes, avec ses imageries propres - Sartre juché sur son tonneau et harangant des ouvriers grévistes -, elle semble appartenir à un autre temps. À défaut de s'engager, les écrivains n'ont toutefois pas renoncé à s'impliquer dans la société. Cette idée d'implication me semble davantage en mesure de définir leur pouvoir d'intervention actuel que la notion caduque d'engagement. Question de perspective: à la figure de l'écrivain engagé est traditionnellement associée une attitude de surplomb par 
l'esprit, de domination par le verbe, de souveraineté par le savoir, un magistère, disait-on naguère; celle de l'écrivain impliqué suppose au contraire la totale immersion de celui qui écrit dans le corps social, une expérience quotidienne de la porosité, sinon de la solubilité, dans une société qui ne devient Cité, c'est-dire ne peut être représentée comme un ensemble identifiable, avec ses caractéristiques saillantes, saisie dans ses évolutions les plus visibles comme dans ses valeurs sous-jacentes, que si elle est pleinement éprouvée par le biais de situations communes. C'est parce qu'il sont rendus à un anonymat relatif que les écrivains peuvent proposer une figuration, une vision, un imaginaire singuliers de leur société. Plusieurs modèles d'intervention possibles se dégagent d'un certain nombre d'oeuvres et suscitent un certain type de textes, appellent un travail littéraire, comme se développent plusieurs figures nouvelles de l'autorité actuelle des écrivains - au sens premier du terme, ce qui fait l'auteur, autorité qui n'est pas arrogance mais voix singulière par laquelle il se qualifie. Quelques figures parmi d'autres, mais plus que d'autres peut-être, me semblent représentatives de ces nouvelles formes d'autorité: celle du témoin en situation, qui par l'écriture se fait aussi, parfois, partisan; celle du conteur, entretenant par l'imaginaire notre sens de l'éthique; celle du passeur, qui, en transmettant sa mémoire des oeuvres, ses lectures, interroge aussi les systèmes de savoir et de communication actuels.

Figure du témoin en situation, tout d'abord: le témoin est celui qui assiste à, qui atteste de, mais aussi dispose, oriente, interprète. Il peut se vouloir un témoin de l'ordinaire, qui renseigne sur les pratiques collectives, les modes de vie, les automatismes du temps, les contrastes culturels, pour peu qu'à l'image d'Annie Ernaux dans ses journaux "extimes", il le consigne et le décrypte simultanément, en prélève des segments et les inscrit dans un dispositif qui fait sens politiquement ${ }^{1}$. Ainsi expérimente-t-elle une écriture mixte, "ethnotexte" dit-elle, qui déplace les marges de la littérature vers celles de la sociologie, comme avec elle l'espace de l'écrivain se déplace de Paris - la capitale, ville fétiche des romanciers - vers la banlieue - la périphérie - à l'image du décentrement culturel, du glissement de terrain symbolique, que connaît la littérature elle-même. Attitude proche, mais levée en fiction et filtrée par l'ironie, chez un écrivain comme Jean Echenoz qui, dans plusieurs de ses romans, excelle à composer, depuis son acuité à l'infra-ordinaire, formule empruntée à Perec, les premiers portraits satiriques de nos temps de société-spectacle. Ces témoins de l'ordinaire se veulent aussi, parfois, témoins d'exception: ainsi du travail mené dans les années 1990 par Hervé Guibert depuis l'expérience de sa maladie, expérience littéraire tout aussi bien puisque le texte se lit à la fois comme un document pleinement clinique, qui expose les différentes phases de développement d'un sida à des fins de révélation publique, mais aussi comme une oeuvre pleinement romanesque, la fiction de soi,

\footnotetext{
${ }^{1}$ Annie Ernaux, Journal du dehors, Gallimard, 1985.
} 
l'autofiction constituant la seule approche possible d'une maladie dont alors on ignore tout. C'est aussi parfois en considérant le fait divers non plus comme un événement hors norme, qui fait sensation, mais comme une situation on ne peut plus commune, comme le basculement potentiel de tout état supposé normal en une crise meurtrière, que d'autres écrivains comme Emmanuel Carrère, avec L'Adversaire, interrogent la vulnérabilité des structures de société et du principe de civilisation: de quels monstres si apparemment dociles, si étonnament conformes à nous-même au point qu'on ne les voit pas notre monde accouche-t-il'²? Témoins d'exception, aussi, tel Jean Hatzfeld avec sa trilogie rwandaise, Dans le nu de la vie, Une saison de machettes, La Stratégie de l'antilope, qui collecte et entrelace des récits de rescapés et des tueurs, les voix des victimes tutsis et des bourreaux hutus $^{3}$. Témoins de l'impossible, pour d'autres, adeptes d'une littérature spectrale: ils écrivent, à la manière de Modiano, depuis une mémoire qui précède leur naissance, celle des années sombres, guerres mondiales ou coloniales, déportations, dont ils se font les intercesseurs, inventant des scènes de hantise, une fois passé le temps des témoins réels, ceux de la première génération. Exemple symptomatique: deux romans dont on a beaucoup parlé beaucoup fin 2009 sont justement des romans qui n'ont rien de saisonnier mais se fondent comme réappropriation d'une mémoire collective et d'une histoire internationale dont leurs auteurs, tous deux nés en 1967, entendent témoigner en tant que ses héritiers directs, ses dépositaires en âge second: Yannick Haënel, avec Jan Karski, interroge la figure même d'un des témoins du film de Claude Lanzmann, Shoah; Laurent Mauvignier, avec Des Hommes, reconstitue la mémoire de la Guerre d'Algérie en partie refoulée par ceux qui l'ont vécue, dans sa propre famille, la génération de son père ${ }^{4}$.

Ces scènes de cauchemar, on les trouve aussi dans des oeuvres qui puisent aux sources de la littérature, réinvestissant la puissance tragique des mythes ou régénérant celle, cruelle, des légendes immémoriales. La figure de l'écrivain-témoin le cède alors à celle de l'écrivain conteur. L'oeuvre de Volodine se développe ainsi comme la mémoire-épouvante des chaos totalitaires du siècle dernier, celle d'Assia Djebar, dans son entrelacs de contes, de séquences historiques et de scènes d'actualité, comme la mémoire convulsive du fait colonial, de ses débuts - la prise de l'Algérie -, à sa fin - la décolonisation -, mais aussi à ses séquelles -les années noires de la toute fin du XXe siècle. Quant à un Pierre Michon ou une Linda Lê, un Pierre Bergounioux ou un Patrick Chamoiseau, ils jouent tantôt du registre de la légende, tantôt de celui de l'épopée pour mettre en scène la mémoire des filiations qui

\footnotetext{
${ }^{2}$ Emmanuel Carrère, L'Adversaire, POL, 2002.

3 Jean Hatzfeld, Dans le nu de la vie, Point Seuil, 2000; Une saison de machettes, 2003; La Stratégie des antilopes, Point Seuil, 2007.

${ }^{4}$ Laurent Mauvignier, Des hommes, Minuit, 2009. Yannick Haenel, Jan Karski, Gallimard, 2009. On se reportera aussi à l'entretien oral accordé par Yannick Haenel à Marc Dambre dans le cadre du séminaire doctoral du Ceracc, animé par Marc Dambre et Bruno Blanckeman, à l'Université de la Sorbonne Nouvelle Paris III, et enregistré par radio Isorbonne (enregistrement accessible sur le site de cette université, entrée "isorbonne").
} 
se perdent, qu'elles s'enracinent dans une France rurale, la Creuse, le Cantal, ou un empire colonial, le Cambodge, les Antilles, la première en déshérence, le second rayé de la carte depuis les années 1950-1960. C'est à une seule et même question que les écrivains renvoient la société, celle de ses propres origines, historiques, généalogiques, symboliques, à une époque où la représentation du passé se montre aussi fuyante que la figuration de l'avenir improbable. Pas plus de socle ne garantit notre discours sur l'histoire quand, de millénaires en millénaires, les fondements de celle-ci sont aspirés dans la nuit des temps, dans une impensable préhistoire, pas plus d'assise, donc, ne garantit notre vision de la chose historique que de finalité, théologie ou téléologie, n'en garantit l'acccomplissement, n'en assure l'avenir. La conscience des temps présents s'écrit entre ces deux lignes de fuite temporelles. Cette question des origines, d'autres écrivains-conteurs la posent pourtant en termes de transcendance, comme pour entretenir par les récits, à rebours d'une époque qui s'en est désaccoutumée, un souci spirituel, marqué par son inspiration chrétienne - Sylvie Germain, Frédéric Boyer - ou un sens du sacré détaché de toute religiosité - Le Clézio, Marie Ndiaye. L'art du conte relaie un souci éthique, quelle qu'en soit la couleur dominante, historique, politique ou spirituelle.

Cette inquiétude des origines, la littérature l'éprouve à son corps défendant, dans une société qui perd l'emprise des lettres, lit ailleurs, surfe sur le Net ou s'affriole de blogs. Loin des histoires littéraires, plusieurs écrivains investissent les multiples mémoires du phénomène littéraire. Quand la littérature implose, seuls restent des livres, projetés hors des hiérarchies de genres, d'époques, de disciplines, dont certains ramassent en pilleurs d'épaves les éclats, les rassemblent dans quelque forme nouvelle, en réinventent l'histoire, aux portes de la légende. Pascal Quignard, Philippe Sollers, mais aussi Gérard Macé, Alain Nadaud, Lydie Salvayre incarnent cette figure de l'écrivain-passeur, passeur de signes et de savoirs. C'est un savoir second, syncrétique, personnalisé à l'extrême, qu'ils transmettent aux lecteurs, un savoir lui-même mis en oeuvre, objet de fiction et de rêverie, qui reconstruit par l'illusion des songes et la médiation des récits les bibliothèques de jadis. À la liberté du passeur se mêle parfois la sagacité de l'herméneute: l'écrivain interroge les systèmes de sens, ensembles de signes et jeux de codes sur lesquels les disciplines du savoir et les secteurs en pointe du monde social fondent leurs pratiques. Il s'agit d'interroger les lieux de décision culturels actuellement dominants depuis leurs propres discours et de soumettre à la question littéraire la langue du "communicationnel", de l'"entrepreneurial", des courriels et SMS, tout ce qui relève des "novlangues" et autres "médialectes" (lire à cet égard les expérimentations narrativo-discursives de Jean-Charles Massera). II s'agit aussi d'affirmer la puissance créatrice de l'écrit quand les pratiques de l'époque privilégient l'image sur les mots, quand l'iconosphère tend à supplanter la logosphère. Un écrivain comme Pascal Quignard ne cesse ainsi d'établir des liens et de susciter une effervescence de pensée entre 
des disciplines dont la spécialisation scientifique se gagne au prix de la sectorisation culturelle, de faire pression sur elles pour comprendre comment de nouvelles anthropologies se découpent, de nouveaux imaginaires de l'être au monde sont en attente de forme pour les énoncer et les comprendre.

Un Nobel de littérature, quel que soit le regard détaché que l'on porte sur tout hochet, exerce une fonction fédératrice. II rassemble des lecteurs de tous horizons autour d'une oeuvre et cet effet tout littéraire, tout laïc, de communion me semble particulièrement manifeste dans la popularité de le Clézio. Est-ce un hasard si celui-ci nous entretient de la question de la communauté difficile, sinon impossible, qui est l'un des motifs obsessionnels de la littérature actuelle, et cela depuis Blanchot, depuis les lendemains de la Seconde Guerre mondiale? Combien d'écrivains évoquent, d'une façon ou d'une autre, une société brisée en autant d'îlotes, une humanité disséminée en autant de solitaires...Opéra muet de Sylvie Germain en constitue au plus près de nous le conte le plus intense. C'est dans le rapport à la langue que se noue cette problématique, parce qu'elle constitue le lien premier qui rassemble ou exclut, la force commune qui unifie ou désagrège la communauté, parce qu'il n'est d'identité, personnelle ou collective, que par les mots que l'on emploie au quotidien pour parler de soi à l'autre, entretenir en soi la conscience de l'autre, tenir ces propos et échanger ces récits au travers desquels notre personnalité se construit à l'échelle d'une vie avec les autres. Cette langue, les écrivains évoqués la travaillent comme un lieu de tensions entre des discours incompatibles, de fractures entre des usages distincts, de dialogue assourdi par des effets de polyphonie ambiants, mais aussi, depuis sa propre syntaxe, ses registres composites, son histoire au long court et au verbe haut, comme un lieu de mémoire, de transmission, de partage, d'expérimentation. 